Article

\title{
Nanoencapsulated Boron Foliar Supply Increased Expression of NIPs Aquaporins and BOR Transporters of In Vitro Ipomoea batatas Plants
}

\author{
Juan Nicolas-Espinosa ${ }^{1}\left(\mathbb{D}\right.$, Pablo Garcia-Gomez ${ }^{1}$, Juan J. Rios ${ }^{2} \mathbb{D}$, Abel Piqueras ${ }^{3} \mathbb{D}$, Gloria Bárzana ${ }^{1}$ \\ and Micaela Carvajal ${ }^{1, * \mathbb{D}}$
}

\section{check for}

updates

Citation: Nicolas-Espinosa, J.;

Garcia-Gomez, P.; Rios, J.J.; Piqueras,

A.; Bárzana, G.; Carvajal, M.

Nanoencapsulated Boron Foliar

Supply Increased Expression of NIPs

Aquaporins and BOR Transporters of

In Vitro Ipomoea batatas Plants. Appl.

Sci. 2022, 12, 1788. https://

doi.org/10.3390/app12041788

Academic Editor: Claudio De

Pasquale

Received: 14 December 2021

Accepted: 6 February 2022

Published: 9 February 2022

Publisher's Note: MDPI stays neutral with regard to jurisdictional claims in published maps and institutional affiliations.

Copyright: (C) 2022 by the authors. Licensee MDPI, Basel, Switzerland. This article is an open access article distributed under the terms and conditions of the Creative Commons Attribution (CC BY) license (https:// creativecommons.org/licenses/by/ $4.0 /)$
1 Group of Aquaporins, Plant Nutrition Department, Centro de Edafologia y Biologia Aplicada del Segura (CEBAS-CSIC), Campus Universitario de Espinardo, 30100 Murcia, Spain; jnicolas@cebas.csic.es (J.N.-E.); pablo.garcia.91@csic.es (P.G.-G.); gbarzana@cebas.csic.es (G.B.)

2 Department of Plant Physiology, Faculty of Sciences, University of Granada, 18071 Granada, Spain; jjrios@ugr.es

3 Plant Breeding Department, Centro de Edafologia y Biologia Aplicada del Segura (CEBAS-CSIC), Campus Universitario de Espinardo, 30100 Murcia, Spain; piqueras@cebas.csic.es

* Correspondence: mcarvaja@cebas.csic.es

Abstract: Nanoencapsulation with proteoliposomes from natural membranes has been proposed as a carrier for the highly efficient delivery of mineral nutrients into plant tissues. Since Boron deficiency occurred frequently in crops, and is an element with low movement in tissues, in this work, nanoencapsulated B vs free B was applied to in vitro sweet potato plants to investigate the regulation of B transporters (aquaporins and specific transporters). Additionally, an metabolomic analysis was performed, and mineral nutrient and pigment concentrations were determined. The results showed high increases in B concentration in leaves when B was applied as encapsulated, but also Fe and Mn concentration increased. Likewise, the metabolomics study showed that single carbohydrates of these plants could be related to the energy need for increasing the expression of most NIP aquaporins (NIP1;2, NIP1;3; NIP4;1, NIP4;2, NIP5;1, NIP6;1, and NIP7) and boron transporters (BOR2, BOR4 and BOR7;1). Therefore, the results were associated with the higher mobility of encapsulated B into leaves and the stimulation of transport into cells, since after applying encapsulated $\mathrm{B}$, the aforementioned NIPs and BORs increased in expression.

Keywords: aquaporins; boron; boron transporters; metabolomic analysis; mineral nutrition; in vitro culture; Ipomoea batatas

\section{Introduction}

Sweet potatoes (Ipomoea batatas [L.] Lam.) are considered to be a major food crop worldwide, and it is widely produced and consumed globally. The country with the highest production is China, with approximately $76.07 \%$ of the world's production [1]. Apart from the edible root, sweet potato leaves are also consumed as a leafy vegetable by humans, and the crop is currently widely used for food due to its high yield, drought tolerance, and the ability to grow in different climates and farming systems. The sweet potato is an important crop globally since it provides food rich in proteins, fibers, mineral nutrients such as $\mathrm{K}, \mathrm{P}$, $\mathrm{Ca}, \mathrm{Mg}, \mathrm{Fe}, \mathrm{Mn}, \mathrm{Cu}$, and bioactive molecules as phenolic compounds [2]. Sweet potatoes are able to maintain high yields even in soils deficient in mineral nutrients; however, some nutrients such as $\mathrm{N}, \mathrm{K}$ and $\mathrm{B}$ must be supplemented [3].

Generally, the aim of micropropagation is the rapid clonal multiplication of plants free of diseases and pests. With this technique, many plants are produced, with all of the clones having the genetic characteristics of the original plant [4]. In this way, plants can be grown in a controlled environment free from genetic and weather alterations. Additionally, in vitro culture technology has the advantage of slowing plant growth by controlling 
external conditions [5]. Likewise, the possibility of studying organized tissues such as shoots, meristems, and embryos makes this technique a fundamental tool for metabolomics and transcriptomics assays [6,7].

Plant short-distance cell-to-cell water transport and homeostasis are regulated by membrane transporters such as aquaporins [8]. Aquaporins are classified within major intrinsic proteins (MIPs) and consist in a family of integral membrane proteins characterized by their conservative structure presenting six transmembrane domains formed by $\alpha$-helixes, two reentrant short $\alpha$-helixes, and five interconnecting loops [9]. This family also has a conservative 3D structure, forming an hourglass morphology with a center pore that allows the passage of small molecules. Aquaporins perform a main role to enable facilitated passive transport, down their concentration gradients, of basically water; however, the capacity of some aquaporin isoforms to facilitate the transport of a broad spectrum of small molecules, such as urea, ammonia, silicon, carbon dioxide, hydrogen peroxide, boron, among many others, has been widely studied [10]. It has been proved that the specificity of molecule transport through different aquaporins isoforms is mostly controlled by the steric occlusion that specific residues provide, and some of the widely described important positions are NPA motifs, ar/R selectivity filters, and Froger's positions (FP) [11-13].

The aquaporins are presently classified into five families, such as PIPs (plasma membrane intrinsic proteins); TIPs (tonoplast intrinsic proteins); NIPs (nodulin-26-like intrinsic proteins); SIPs (small intrinsic proteins); and XIPs (uncharacterized intrinsic proteins [14]. In particular, among the entire family of aquaporins, the NIP subfamily is the one that can transport the greatest variety of molecules [15]. In particular, several NIPs aquaporins have been reported to facilitate the diffusion of $B$, in the form of boric acid, across the biological membranes. It has been shown that in the case of B deficiency conditions a reduction of water transport from the root to the aerial part was observed, and an associated increase in aquaporin NIP5;1 and NIP6;1 expression in A. thaliana plants [16].

Boron is also mobilized by an active process carried out by specific transporters located in membrane cells belonging to BOR family, including BOR1 and BOR2 groups [17]. In the same way, like NIPs aquaporins, the expression of B transporters, BOR1 and BOR2, was regulated for maintaining plant and cell $\mathrm{B}$ concentration and was significantly upregulated under B starvation [18]. It has been reported that B can be transported through plants by an active process mediated by the specific transporter BOR1 localized in membrane cells [17]. In addition, some aquaporins—such as NIP5;1—were observed to be able to transport B [19]. Additionally, the deficiency of B leads to a reduction in water transport from root to leaf, and an associated increased in NIP5;1 and NIP6;1 expression in A. thaliana plants [16]. The increases in the expression of other NIPs aquaporins, such as NIP3, 1 in rice and NIP2,1 in barley, have been associated with B transport, suggesting the involvement of NIPs aquaporins in B uptake and transport [20,21]. In the same way, the expression of $\mathrm{B}$ transporters, BOR1, and BOR2, was regulated for maintaining plant and cell B concentration [18]. Likewise, $\mathrm{B}$ has been reported to reach long distance from leaves to roots via phloem, by the B-sucrose complex [22].

The nanoencapsulation of boron has been applied to almond trees [23,24], demonstrating that the efficiency in B transport into tissues was much higher than when free B was applied. In fact, a higher nutrient penetration through the leaf cuticle was demonstrated [23] by the fact that nanocapsules containing fluorescein disodium salt (FNA) were able to penetrate into leaf tissues in $2 \mathrm{~h}$ whereas no penetration was observed in free form. Additionally, they were able to penetrate and reach other tissues separated from those where B was applied. Likewise, nanoencapsulation with proteoliposomes from natural membranes has been shown to behave as an effective carrier [25], as these were able to deliver mineral nutrients into cells [26].

Therefore, the aim of this work was to establish the regulation of B transporters (aquaporins and specific transporters) when boron concentration increased in leaves. We determined the changes in metabolism that could be associated with the regulation in these transporters. For this, different applications of free B and encapsulated B were 
subjected to plants, then measurements of mineral nutrients, pigment concentration, and a metabolomic analysis were associated to the expression of NIP aquaporins and BOR transporters. We expected to find that primary metabolite changes, in conjunction with an increased expression of aquaporins, was a method to determine the possible connections.

\section{Materials and Methods}

\subsection{Plant Growth}

The plants were obtained from micropropagated sweet potato (Ipomoea batatas, [L.] Lam) shoot cultures, grown on a solid Murashige and Skoog (MS) medium supplemented with $60 \mathrm{mg} \mathrm{L}^{-1}$ phloroglucinol, $30 \mathrm{mg} \mathrm{L}^{-1}$ sequestrene (DDHA-Fe, $6 \% \mathrm{Fe}$ ), $0.15 \mathrm{mg} \mathrm{L}^{-1}$ meta-topolin, $6 \mathrm{mg} \mathrm{L}^{-1}$ adenine sulfate, $0.040 \mathrm{mg} \mathrm{L}^{-1}$, indole butyric acid, $3 \%$ sucrose, and at $\mathrm{pH}$ of 5.8. Shoot cultures of sweet potato were subcultured as nodal segments every four weeks.

For this experiment, shoots with three internodes and fully expanded leaves were transferred to $1 \mathrm{~L}$ vitrovent (Duchefa, Haarlem, The Netherlands) culture containers (one plant each), which contained $150 \mathrm{~mL}$ of $1 / 2 \mathrm{MS}$ medium without growth regulators, containing $40 \mathrm{mg} \mathrm{L}^{-1}$ sequestrene, $80 \mathrm{mg} \mathrm{L}^{-1}$ phloroglucinol, $250 \mathrm{mg} \mathrm{L}^{-1}$ MES buffer, $0.7 \%$ Agar, and at $\mathrm{pH}$ of 5.8. Under these conditions, the shoots elongated, developed fully expanded leaves, and rooted in 6 weeks. All the cultures were maintained at $25 \pm 2{ }^{\circ} \mathrm{C}$ in a growth chamber with a $16 \mathrm{~h}$ photoperiod $\left(80 \mu \mathrm{mol} \mathrm{m} \mathrm{m}^{-2} \mathrm{~s}^{-1}\right.$ photosynthetically active radiation, PAR).

The treatments applied were (i) B-nanoencapsulated at $0.04 \% w / v\left(\right.$ from $\mathrm{H}_{3} \mathrm{BO}_{3}$ ) obtained according to Rios et al. (2020) [23]; (ii) free $\mathrm{B}$ at $0.04 \%$ (from $\mathrm{H}_{3} \mathrm{BO}_{3}$ ), with the control plants treated with water only. The treatments were applied only to the abaxial side of all leaves in each plant using a paintbrush. Furthermore, the treatment solutions contained a surfactant (polyether-modified-polysiloxane) at a concentration of $0.1 \% v / v$ to improve the contact between the surface of the leaves and the solutions. In addition, and according with results demonstrated by Rios et al. 2019, the nanovesicles themselves did not produce any effects on the plants. The sample collections were taken 2 days after treatment application.

\subsection{Analysis of Mineral Nutrients}

For ion analysis, leaves were collected, dried, and ground into a fine powder. Then, the samples were digested in a microwave oven (CEM Mars Xpress, Stallings, NC, USA), by HNO3:HClO4 (2:1) digestion. The elements were detected by inductively coupled plasma (ICP) analysis (Optima 3000, PerkinElmer, Waltham, MA, USA).

\subsection{Pigment Analysis}

Pigment analyses were carried out as proposed by Arnon (1949) [27] with some modifications. Plant leaves were collected, and $3 \mathrm{~mm}$ pieces were trimmed to obtain $40 \mathrm{mg}$ of each biological sample. Then, $5 \mathrm{~mL}$ of methanol was added into $10 \mathrm{~mL}$ tubes and sealed with parafilm. The samples were stored in darkness overnight and the pigments were measured with a spectrophotometer (Helios alpha, ThermoSpectronic, Cambridge, UK) under various wavelengths $(450,487.5,502,530,645$, and $663 \mathrm{~nm})$. Pigment concentrations were calculated with using the following equations:

$$
\begin{gathered}
\text { Chlorophyll a }(\mathrm{Ca})\left(\frac{m g}{m L}\right)=\left(12.7 \times A_{663}\right)-\left(2.69 \times A_{645}\right) \\
\text { Chlorophyll b }(\mathrm{Cb})\left(\frac{m g}{m L}\right)=\left(22.9 \times A_{645}\right)-\left(4.68 \times A_{663}\right) \\
\frac{\mathrm{Ca}}{\mathrm{Cb}}=\frac{0.457\left(4.7\left(A_{663}-A_{643}\right)\right)}{4.87\left(A_{645}-A_{663}\right)} \\
\text { Carotenes }\left(\frac{m g}{m L}\right)=\left(4.07 \times A_{450}\right)-((0.0435 \times C a)-(0.367 \times C b))
\end{gathered}
$$




$$
\text { Lycopene }(\%)=\frac{\left(\frac{181 \times A_{502}}{A_{487.5}}\right)-42}{237} \times 100
$$

With $A_{x}$ being the absorbance measured for the indicated wavelength represented. The data measured in $\mathrm{mg} \mathrm{mL}^{-1}$ were then transformed to a mg $100 \mathrm{~g}^{-1}$ of fresh weight.

\subsection{Metabolomic Analysis}

To determine polar and semi-polar metabolites, $50 \mathrm{mg}$ of ground and lyophilized leaf samples were placed in $2 \mathrm{~mL}$ Eppendorf tubes. To this tube, $1200 \mu \mathrm{L}$ of a methanolwater mixture was added $\left(\mathrm{MeOH}: \mathrm{H}_{2} \mathrm{O} ; 1: 1\right)$ and vigorously vortexed. The samples were then sonicated three times and incubated for $30 \mathrm{~min}$ at $4{ }^{\circ} \mathrm{C}$. After that, the samples were centrifuged at $11,000 \times g$ for $20 \mathrm{~min}$ at $4{ }^{\circ} \mathrm{C}$. The supernatant was passed to a new $1.5 \mathrm{~mL}$ Eppendorf tube and dried with a speed vacuum overnight to a maximum temperature of $30{ }^{\circ} \mathrm{C}$ to completely dry the samples. Dried samples were resuspended in $800 \mu \mathrm{L}$ of a $100 \mathrm{mM}$ potassium phosphate buffer $\left(\mathrm{KH}_{2} \mathrm{PO}_{4}\right)$ at $\mathrm{pH}=6.0$ (diluted in $\left.100 \% \mathrm{D}_{2} \mathrm{O}\right)+$ $0.58 \mathrm{mM}$ of TSP- $\mathrm{d}_{4}$ (internal standard). After this, the samples were filtered with $0.45 \mu \mathrm{m}$ pore diameter nylon filters. Finally, $600 \mu \mathrm{L}$ of the filtered samples were placed in a $5 \mathrm{~mm}$ NMR tube for their quantification by H-NMR [28,29].

\subsection{Aquaporin and BOR Primers Design and Expression}

For primer design, an approximation strategy was followed, since the genome of Ipomoea batatas is not published and the data are not available; however, information is available for two close relatives, Ipomoea triloba and Ipomoea trifida, with these two species being possible progenitors of Ipomoea batatas [30,31]. For this reason, in order to design suitable primers for sweet potatoes (batata), several aquaporins genes (NIPs) and boron transporters (BOR) were identified in I. triloba and I. trifida, and orthologous genes were paired and aligned to obtain consensus sequences using Geneious Prime 2020.1.2 software (https://www.geneious.com/), (accessed on 8 December 2021). Conserved sites from these consensus sequences were selected as the targets for primer design.

The primer design was carried out manually, meeting the specific requirements imposed by the high homology of the sequences used and the technique used for the analysis. As housekeeping, IbACT, was used [32]. A virtual analysis of melting temperature, primer hairpins, self-dimers, hetero-dimers, and individual and total $\Delta \mathrm{G}$ was performed with PCR Primer Stats (https:/ / www.bioinformatics.org/sms2/pcr_primer_st), (accessed on 10 November 2021) and IDT OligoAnalyzer tools (https:/ / eu.idtdna.com/calc/analyzer/), (accessed on 10 November 2021). The $\Delta \mathrm{G}$ accepted for dimer analysis was less than $-6 \mathrm{kcal} / \mathrm{mol}$. The specificity of the amplicons was checked using the virtual nucleotide basic local alignment search tool (NCBI nucleotide BLAST: https:/ /blast.ncbi.nlm.nih.gov/ Blast.cgi), (accessed on 25 November 2021). The efficiency of the primer sets was evaluated with the software QuantStudio 5 (QuantStudio TM Design and Analysis Software version 1.4.0.0, Waltham, MA USA), by analyzing the threshold cycle (Ct)/fluorescence ratio at six independent points of the PCR curves [33], obtaining values between 95 and $100 \%$. Four housekeeping gene primers [18 ribosomal RNA (18rRNA), elongation factor 1-alpha (EF1a), actin (ACT), and eukaryotic initiation factor 4A (elF4a)] for Ipomoea triloba were selected according to GuoLiang et al. (2020) [32] and checked with each cDNA used in the quantitative PCR (qPCR), after which, these were measured using a Visual Basic Application for Excel (GeNorm), which automatically calculates gene stability [34]. The most stable constitutive gene, ACT, was then selected as the reference gene for standardization. The sequences and features of the primers used for the 11 I. batatas NIP genes, 5 BOR transporters, and one constitutively expressed gene, are shown in Table 1. 
Table 1. Primer set used to perform expression analysis.

\begin{tabular}{|c|c|c|}
\hline Gene Name & Forward $\left(3^{\prime}-5^{\prime}\right)$ & Reverse $\left(3^{\prime}-5^{\prime}\right)$ \\
\hline IbNIP1;1 & CATGCTCTACACCGTCG & GGTCCCAGTTGCTAGAG \\
\hline IbNIP1;2 & GCAAGGAGTTTGGGTCC & CCTGAGGATGTTGTAGACC \\
\hline IbNIP1;3 & TGATGGCAGAGAGTAATGG & GGAGAGTGAAGATGAAATCG \\
\hline $\mathrm{IbNIP} 2 ; 1$ & GGAGAATGAAGACGGAAAGG & TCACAAACACCAACAAATACG \\
\hline IbNIP4;1 & CTGGAGCGTCAATGAACC & TGTGAGTGAGTTTAAGCACG \\
\hline $\mathrm{IbNIP} 4 ; 2$ & CATTCAAAAGATGATTGCTGAG & CATAACCATGATGACCAAACC \\
\hline IbNIP5;1 & AATACGAGGCTGCTAGAGC & CTGGATTCCGATCACACG \\
\hline $\mathrm{IbNIP5;2}$ & GACTGGCAGTTATGATTGTG & ATGGGAAGTGACGAAACG \\
\hline $\mathrm{IbNIP5;3}$ & CGTGTCTCACCGATTTTCC & GCATTCCCAATCAACGACT \\
\hline $\mathrm{IbNIP6;1}$ & AGAAAGGTGGGAGCAGAG & GGTTGACAATGGCTGTGG \\
\hline IbNIP7;1 & TATGATGACAAGACCACTCC & TCCAACAACAAATCCTGC \\
\hline IbBOR1 & CGTGGCATCACAACTTGCTC & TGACACCATTTGATGGCGGA \\
\hline IbBOR1L & CTTGCATGGACTGGATGGGT & ATCCCAAACAGCTCTCCAGC \\
\hline IbBOR2 & TATCGGAGCATGCAAGAAGC & GCACCAATGTGCCCTGTACT \\
\hline $\mathrm{IbBOR} 4$ & ATGGGCTGCTAGGGGTCATA & CTGAACCAGCCTGTGCCATA \\
\hline IbBOR7;1 & CGTCTGCTCTTCCGGTCATT & TGCCGGCAAAAGTGTACAGA \\
\hline $\mathrm{IbACT}$ & TGCCTGATGGACAAGTGA & GGAGCGAGTGCTGTAATT \\
\hline
\end{tabular}

Lastly, the expression level of all the aquaporin genes was measured from $2 \mu \mathrm{L}$ of 1:10 diluted cDNA following the system instructions in the QuantStudio 5 Flex, a Real-Time qPCR system (Applied Biosystems by Thermo Fisher Scientific, Waltham, MA, USA). The qPCR program consisted of a $10 \mathrm{~min}$ initial denaturation at $95{ }^{\circ} \mathrm{C}$ and then amplification in a two-step procedure: $15 \mathrm{~s}$ of denaturation at $95^{\circ} \mathrm{C}$ and $60 \mathrm{~s}$ of annealing and extension at a primer-specific temperature for 40 cycles, followed by a dissociation stage. Real-time PCR measurements were carried out in four independent RNA samples per treatment (biological replicates), and the $\mathrm{Ct}$ was determined in triplicate (technical replicates) in 96-well plates. Negative controls without cDNA were used in all the PCR reactions. The transcript levels were calculated using the $2^{-\Delta \Delta \mathrm{Ct}}$ method [35]. Finally, the normalized expression levels were rescaled and presented as relative unit expression (r.u) with respect to the control expression, which was assigned a value of 100 .

\subsection{Statistical Analysis}

Statistical analyses were carried out using SPPS 25.0.0.1. software. Significant differences were calculated with a one-way ANOVA and Duncan algorithm post hoc with a $95 \%$ confidence level and a $p<0.05$. All the data are represented as means \pm standard error (SE).

\section{Results}

The results of the mineral nutrient analysis of leaves (Figure 1) revealed a general maintenance of macronutrients (Figure 1a) and micronutrients (Figure 1b) of I. batatas leaves after the treatments were applied. 
a)

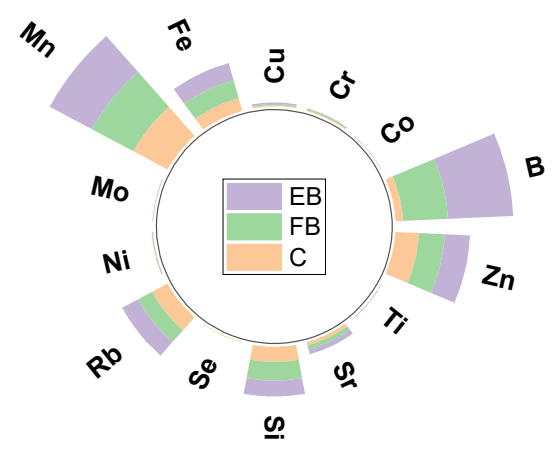

c)

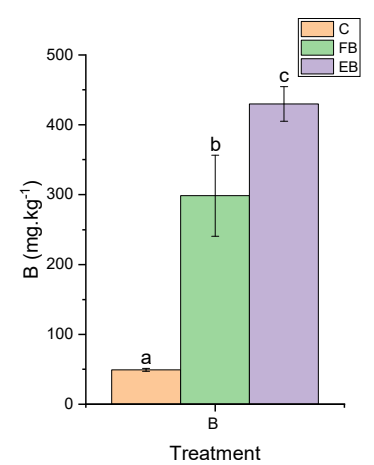

d)

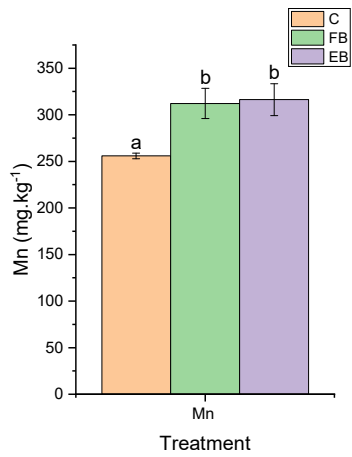

b)

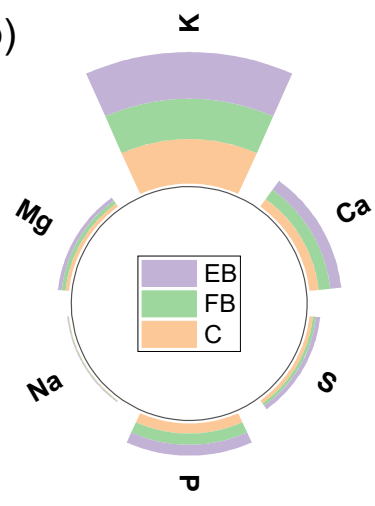

e)

Figure 1. Analysis of mineral nutrients in I. batatas leaves. (a) Radial stacked bar representing relative quantities of macronutrients presented in I. batatas leaves of control (C), free boron (FB) and encapsulated boron (EB) treatments. (b) Radial stacked bar representing relative quantification of some micronutrients. (c) Boron quantification in $\mathrm{mg} \cdot \mathrm{kg}^{-1}$ of dry mass in I. batatas leaves. (d) Manganese quantification in $\mathrm{mg} \cdot \mathrm{kg}^{-1}$ of dry mass in I. batatas leaves. (e) Iron quantification in $\mathrm{mg} \cdot \mathrm{kg}^{-1}$ of dry mass in I. batatas leaves. Different letters in (c-e) mean statistical differences $p<0.05$.

In particular, only $\mathrm{B}, \mathrm{Fe}$, and $\mathrm{Mn}$ changed after the treatment application. The rest of the nutrient did not show significant differences between the control plant leaves and any of the B treatments (free and encapsulated). The concentration of B (Figure 1c) showed a significant increase when free B was applied, although this increase was higher with encapsulated B, with almost ten times greater concentrations of B in leaves observed when directly compared with control plants leaves, according to Duncan algorithm $p<0.05$. Likewise, significant differences also appeared between the free $B$ and encapsulated $B$ treatments, being 1.5-fold higher in encapsulated B treatments. Additionally, Mn (Figure 1d) and $\mathrm{Fe}$ (Figure 1e) showed significant increases after B application. However, the values did not significantly differ between the B treatments.

Additionally, the analysis of the chlorophyll a and b, carotenoids, and lycopene pigments (Figure 2) revealed the highest concentration of chlorophyll a as compared to the other pigments, although the concentration of chlorophyll b, carotenoids, and lycopene (as part of the carotenoids) was similar in control and B-treated plant leaves. Overall, no differences were observed between plant leaf pigments among treatments. 


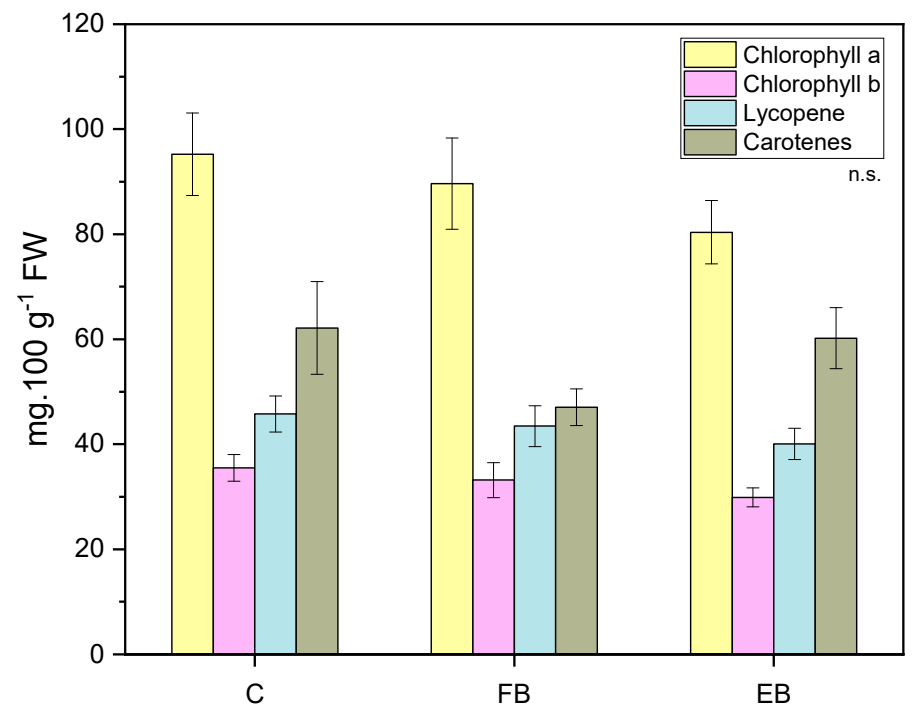

Figure 2. Analysis of pigments in I. batatas leaves of the different treatments (C, control; FB, free boron; EB, encapsulated boron). Data are represented as means and SE errors. No statistical differences were observed (n.s.).

The metabolomics analysis (Table 2) showed that organics such as citric acid, formic acid, and malic acid appeared in relatively high concentrations. However, only formic acid showed a significant increase and only after encapsulated B was applied. The determination of amino acids showed that only asparagine and valine showed significant increases when encapsulated B was applied, but no significant differences appeared between control and free B. It was also observed that asparagine was the amino acid with the highest concentration, being 10 times higher than arginine and glutamine.

Table 2. Primary metabolites from sweet potato leaves after 3 days of treatment application. Data are shown as concentrations in $50 \mathrm{mg}$ of sample. Data are expressed as means \pm SE. Different letters refers to statistical differences calculated with ANOVA and Duncan test as a post hoc test $(p<0.05)$.

\begin{tabular}{cccc}
\hline & & Treatments & \\
\hline Metabolites [mM] & C & FB & EB \\
\hline Citric acid & $0.800 \pm 0.286$ & $0.440 \pm 0.149$ & $0.953 \pm 0.334$ \\
Formic acid & $8.283 \pm 0.707 \mathrm{a}$ & $5.777 \pm 0.656 \mathrm{a}$ & $14.490 \pm 1.643 \mathrm{~b}$ \\
Malic acid & $2.380 \pm 0.882$ & $0.970 \pm 0.216$ & $2.680 \pm 1.097$ \\
Alanine & $0.960 \pm 0.182$ & $0.620 \pm 0.029$ & $0.910 \pm 0.208$ \\
Arginine & $8.533 \pm 0.757$ & $7.980 \pm 0.723$ & $8.220 \pm 0.730$ \\
Asparagine & $19.850 \pm 2.158 \mathrm{a}$ & $36.583 \pm 3.704 \mathrm{~b}$ & $43.107 \pm 6.709 \mathrm{~b}$ \\
Glutamine & $3.847 \pm 1.178$ & $6.020 \pm 1.075$ & $6.017 \pm 1.021$ \\
Isoleucine & $0.167 \pm 0.009 \mathrm{~b}$ & $0.107 \pm 0.012 \mathrm{a}$ & $0.187 \pm 0.017 \mathrm{~b}$ \\
Leucine & $0.180 \pm 0.021$ & $0.137 \pm 0.012$ & $0.197 \pm 0.041$ \\
Phenylalanine & $0.203 \pm 0.032$ & $0.163 \pm 0.026$ & $0.257 \pm 0.048$ \\
Threonine & $0.510 \pm 0.021 \mathrm{~b}$ & $0.467 \pm 0.033 \mathrm{a}$ & $0.593 \pm 0.064 \mathrm{~b}$ \\
Tryptophan & $0.387 \pm 0.044$ & $0.323 \pm 0.074$ & $0.443 \pm 0.050$ \\
Tyrosine & $0.133 \pm 0.009$ & $0.083 \pm 0.019$ & $0.133 \pm 0.034$ \\
Valine & $0.220 \pm 0.012 \mathrm{a}$ & $0.197 \pm 0.017 \mathrm{a}$ & $0.313 \pm 0.052 \mathrm{~b}$ \\
Fructose & $2.783 \pm 0.752 \mathrm{a}$ & $2.993 \pm 0.908 \mathrm{a}$ & $8.000 \pm 1.631 \mathrm{~b}$ \\
Glucose & $2.040 \pm 0.517 \mathrm{a}$ & $3.040 \pm 0.364 \mathrm{a}$ & $5.687 \pm 1.310 \mathrm{~b}$ \\
Myo-inositol & $0.277 \pm 0.078$ & $0.410 \pm 0.191$ & $0.650 \pm 0.120$ \\
Sucrose & $1.847 \pm 0.247 \mathrm{~b}$ & $2.163 \pm 0.353 \mathrm{~b}$ & $0.473 \pm 0.046 \mathrm{a}$ \\
UDP glucose & $0.033 \pm 0.003$ & $0.037 \pm 0.003$ & $0.030 \pm 0.006$ \\
Betaine & $0.680 \pm 0.095$ & $0.433 \pm 0.071$ & $0.540 \pm 0.117$ \\
Choline & $0.670 \pm 0.110$ & $0.470 \pm 0.062$ & $0.697 \pm 0.162$ \\
\hline
\end{tabular}


As for the sugars (Table 2), glucose, fructose, and myo-inositol were significantly higher only in plants treated with encapsulated B. However, when sucrose was determined, the opposite effect was observed. In this case, a significant decrease appeared in encapsulated $B$ treated plants. As for the concentration of UDP-glucose, no significant differences were observed. Similarly, the metabolites betaine and choline appeared in low concentrations, but no changes in their concentrations were observed after the application of the treatments.

Phylogenetic analyses (Figure 3) revealed an almost pair-to-pair homology between protein sequences of the two Ipomoea species (I. triloba and I. nill) used as templates to design primers in order to analyze the expression of the NIP aquaporin family in Ipomoea batatas. The NIP family presents a high homology between species, being more evident within the I. nill, I. triloba, and A. thaliana species whose protein sequences cluster in the same clades in almost all NIPs isoforms. On the contrary, as was expected, the two cereal species Z. mays and O. sativa showed a high homology between their isoforms. As revealed by the phylogenetic tree (Figure 3), NIPs aquaporins of Ipomoea species are divided into seven groups from NIP1 to NIP7, except NIP3, which only appeared in I. triloba plants.

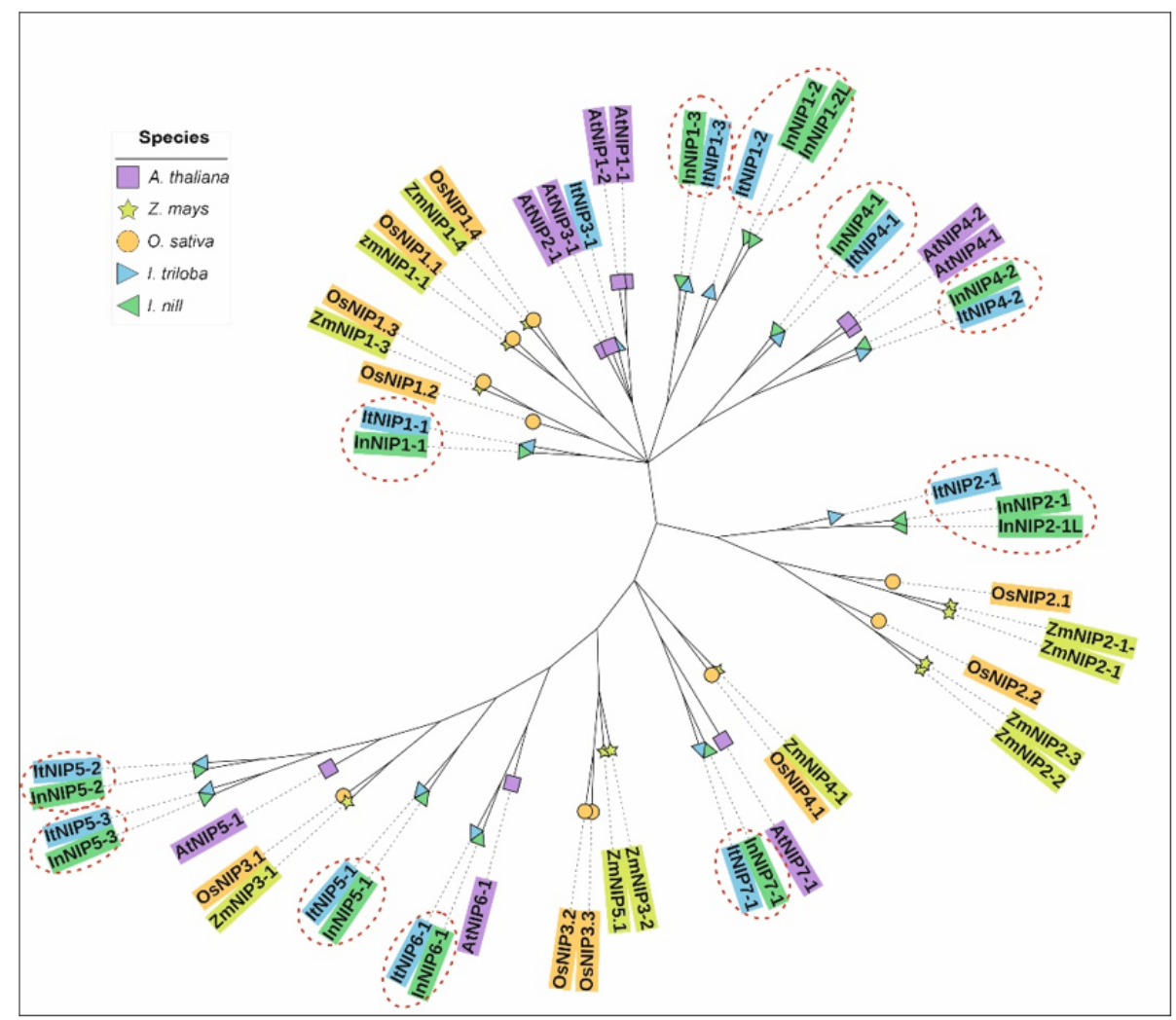

Figure 3. Phylogenetic analysis of NIP aquaporin family protein of A. thaliana, Z. mays, O. sativa, I. triloba and I. nill. MUSCLE algorithm and NJ method (1000 bootstrap replicates) were used in multiple alignments and tree construction respectively. Tree formatting and representation was made with the online tool "Interactive Tree Of Life" (iTOL; https://itol.embl.de/), (accessed on 3 February 2022). Red circles represent the homology groups of sequences used as template to design I. batatas primers.

The relative expression of genes related to B uptake, such as NIP aquaporins and BOR transporters genes, (Figure 4) revealed a general increase of those in plant leaves treated with nanoencapsulated B. More specifically, it can be observed that aquaporins NIP1;2, NIP1;3; NIP4;1, NIP4;2, NIP5;1, NIP6;1, and NIP7;1 (Figure 4B,C,E-G,J,K) were significant increased only in those plants treated with encapsulated $B$, whereas those treated freely with B did not change their expression as compared with the controls. On the contrary, the expression of NIP5;2 and NIP5;3 did not change in any of the treatments (free or 
encapsulated), as compared with the controls. Particularly, only NIP1;1 expression was affected when free $B$ was applied, with statistical differences observed when compared with the control, although no differences were observed when compared with nanoencapsulated $B$ treated leaves.
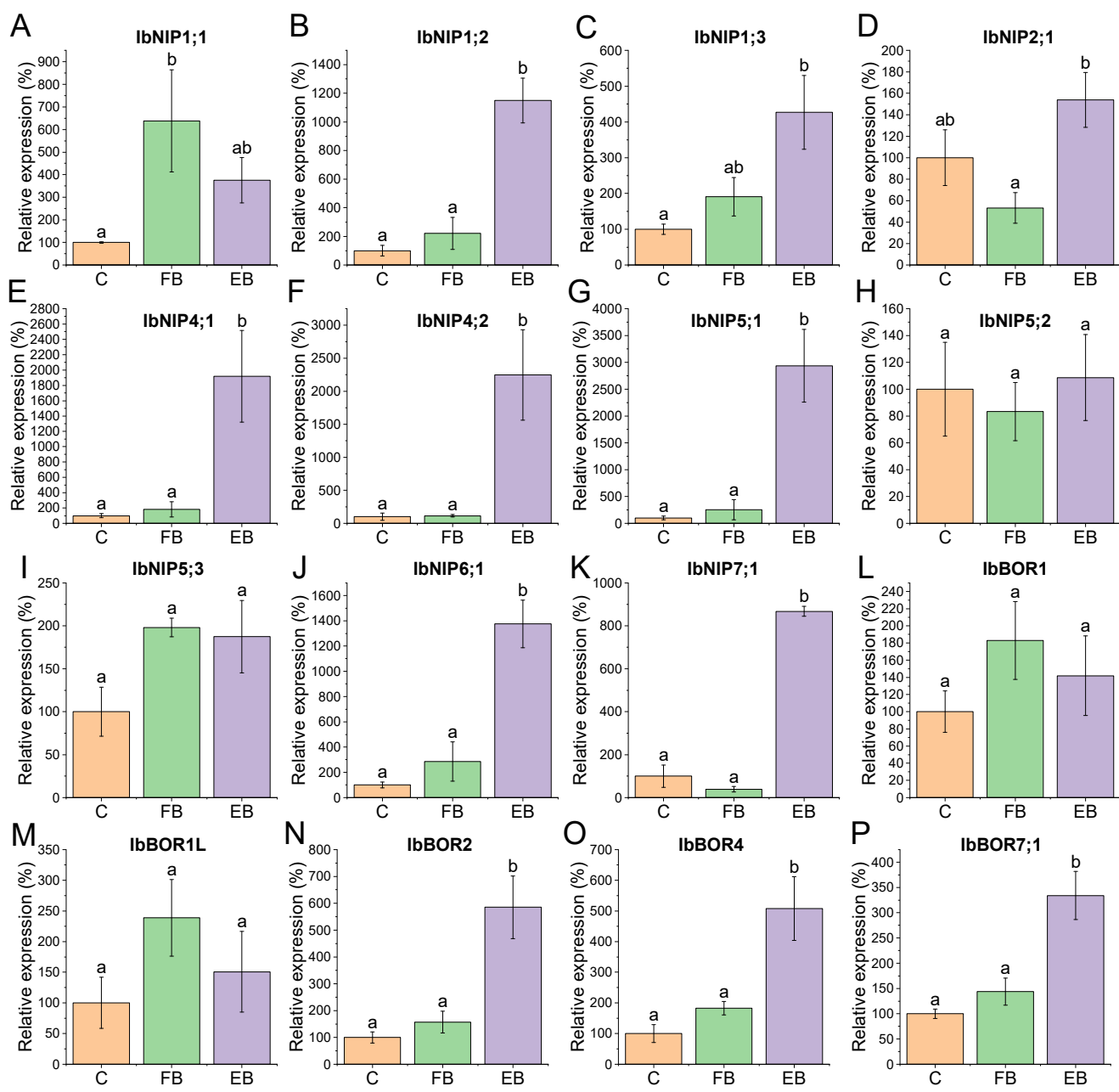

Figure 4. Expression analysis by quantitative PCR of NIP aquaporin and BOR genes from I. batatas leaves (C, control; $\mathrm{FB}$, free boron; $\mathrm{EB}$, encapsulated boron). From (A-K), inclusive, data relative to NIPs aquaporins expression, being, (A): IbNIP1;1, (B): IbNIP1;2, (C): IbNIP1;3, (D): IbNIP2;1, (E): IbNIP4;1, (F): IbNIP4;2, (G): IbNIP5;1, (H): IbNIP5;2, (I): IbNIP5;3, (J): IbNIP6;1, (K): IbNIP7;1. Additionally, from (L-P) data relative to BOR genes expression ((L): IbBOR1, $(\mathbf{M})$ : IbBOR1L, $(\mathbf{N})$ : IbBOR2, (O): IbBOR4 and (P): IbBOR7;1). Statistical analysis was performed using SPSS 25.0.0.1. The values are the means \pm SE of relative expression been $100 \%$ the control plants, different letters show statistical differences $(p<0.05)$.

Moreover, according to the $\mathrm{B}$ transporters, the relative expression of BOR1 and BOR1L was not altered by any of the treatments, although the relative expression of BOR2, BOR4 and BOR7;1 again increased greatly only in the encapsulated B treatment, whereas in the free $B$ treatment, their expression did not change as compared to the control.

\section{Discussion}

Boron has been reported to be transported passively, driven by the transpiration stream when an optimal supply was provided [36]. In the same manner, B has been shown to enter cells by diffusing through the plasma membrane with no active transport, likely facilitated by channels. According to the B concentration found in the leaves of sweet potatoes tested in the present study, it was observed that it was higher after B application, but to 
a higher extent when B was supplied in a nanoencapsulated form. This effect has been previously observed in almond trees [24], but these plants were B deficient. The greater penetration and mobility of nanoencapsulated B as compared to the free form observed in previous studies, was related to a higher ease of penetration through the epidermis facilitated by nanovesicles [23]. It was observed that penetration should have occurred through stomatal pores and aqueous pores, producing a greater distribution within the leaf tissues. However, B was observed to penetrate and reach other tissues only when it was encapsulated, producing physiological changes such as an increase in gas exchange, and root hydraulic conductance in relation to the higher expression of aquaporins that transport water. Thus, the experiments conducted in the present study with in vitro cultures of Ipomoea expand our knowledge on the mechanism of action of encapsulated B. In this way, as water transport is very low in these in vitro plants, the movement of encapsulated $B$ must use other mechanisms to get B into foliar leaf tissue, which should account for the movement of the nanovesicles in tissues.

Nanofertilizers are starting to be used in crop nutrition via soil or/and via foliar applications, providing a faster and higher transport to different parts of plants [37]. After penetrating the leaf or root cuticle tissue, nanofertilizers can be transported through different parts of the plant, which characterize their effectiveness and final destination [38]. In our experiments, the boron concentration was much higher in leaves when it was encapsulated, demonstrating that penetration was more effective. However, the increase in $\mathrm{Mn}$ and Fe was also observed, although it was similar in both B treatments. The application of micronutrient fertilizers has been reported to be a promising strategy for the biofortification of crops [39]. In this way, micronutrient fertilizers can be applied to leaves for improving the concentration and bioavailability of different micronutrients. However, the connection between micronutrients is unknown, and recent studies only showed the concentration due to the enrichment of the micronutrient that was supplied [40]. In this way, the recent investigation in the regulation of micronutrient transporters revealed that plants must have a fine and flexible system to deal with the alteration of the environment for acquiring the specific level of minerals for completing the cellular cycle. In this way, some reports revealed that nutrient transporters are regulated (transcriptionally, translationally, and/or post-translationally) when changes in nutrients occurred. For example, it has been observed that the expression of Fe transporter, AtIRT1, was increased when Fe was deficient [41]. However the amount and localization of the protein, AtIRT1, was not dependent on the supply of Fe [42]. Additionally, the mRNA of a AtNIP5;1, an aquaporin that transports B increased its expression under B deficiency [43], but protein was reduced under the excess of B in Arabidopsis [44]. Interestingly, some nutrient transporters were not related to the availability of mineral elements when transcriptional or post-transcriptional regulation was determined. In this way, low or high Mn availability did not affect the expression of Mn transporter, OsNramp5, in rice [45]. Nevertheless, another Mn transporter, OsNramp3, was reported to have controlled Mn distribution, which changed to an open or closed state, as Mn is needed in specific tissues. [46].

In addition, it is necessary to emphasize that $\mathrm{Fe}$ and $\mathrm{Mn}$ concentrations in leaves increased in both B treatments (free and nanoencapsulated) when compared with the control, but no differences were observed between them. This could be explained by the interaction between nutrients and applied B, inducing the uptake of some nutrients such as $\mathrm{Mn}$ and Fe [47]. Furthermore, B sprayed as a foliar application on Arachis hypogaea, groundnut plants, showed an increase in the uptake of N, P, K, Fe, and Mn [48]. On the contrary, only changes in Fe and $\mathrm{Mn}$, apart from B, were observed in our study. This could be explained by the activation of some routes by B accumulation in leaves, which activated divalent cation transport, as these nutrients could be mobilized by the same type of transporters. For example, Arabidopsis AtNRAMP genes can be found which are involved in the mobilization of both Fe and Mn [49]. Therefore, the homeostasis of the micronutrients is still unknown, although the mechanisms controlling the expression of transporter genes in response to the fluctuation of mineral elements, and the mechanisms 
controlling the polarity of transporters, are being investigated [50]. The fact that the external $\mathrm{B}$ application to non-deficient $\mathrm{B}$ sweet potato plants changed the concentrations of $\mathrm{Fe}$ and $\mathrm{Mn}$ in leaves should provide clues about the inter-regulation of different transporters.

The metabolomic analysis revealed that formic acid increased only in plants treated with encapsulated B. The formation of formiate from glyoxylate has been associated to synthase reactions of glyoxylate and succinate, and the conversion of serine to pyruvate [51]. In this way, the function of the Krebs cycle and mitochondria were analyzed, together with the significance of energy dissipation in the peroxisomes for the regulation of the cell energy balance, with the conclusion that the main function of the photorespiratory peroxisomes was associated with the integration of metabolite flows in the photosynthesizing plant cell. If formic acid is related to energy needs, it could be associated to the changes in the concentration of carbohydrates. The finding that the concentrations of fructose and glucose increased in these plants, although the concentration of sucrose decreased, implies a direct need to obtain energy, but no changes in chlorophylls and carotenoids were observed in any of the treated plants. Since it has been reported that water regulation is related to abscisic acid, which strongly up-regulates the gene expression and enzymatic activities that accelerate water soluble carbohydrates remobilization [52], this could be related to our metabolomics results, given the importance of carbohydrate remobilization in sweet potato plants [53]. Therefore, these metabolic changes could be associated with the changes in aquaporins that react directly to B supplementation.

The NIPs aquaporins have been reported to play a main role in the transport of metalloids, such as B, Si, As, and Sb [54]. NIPs were initially described as glycerol transporters, but their similar conformation and charge with other metalloid compounds provided evidence of transport activities [55]. Twelve NIPs have been found in Ipomoea batatas, which were classified into the seven different subgroups of NIPs based on their ar/R filter and homology [15]. Our expression predictions, supported by the results shown in Figure 4, and the identification of residues key for B transport (Supplementary Table S1) suggested that all the aquaporins described in this work could transport B, aside from water (Table 1). However, in most of the studies found, the increase in NIP expression occurred under B deficiency. Accordingly, under B deficiency, damage to the xylem reduced water transport from roots to leaves, thereby affecting the distribution of other elements. Therefore, the expressions of aquaporins NIP5;1 and NIP6;1 were reported to highly increase $[43,56]$. Likewise, the expression of NIP3;1 in rice and NIP2;1 in barley (Hordeum vulgare L.) were described as B transporters $[20,21]$. However, the fact that in our experiments the B concentration was optimal implied that the B transport through NIP aquaporins was induced when transport of $\mathrm{B}$ through tissues was stimulated by higher boron concentrations.

Lastly, under B toxicity, BOR1 has been reported to be degraded in order to reduce the transport of B from root to shoot [18]. In this study, both BOR1 isoforms were the only ones that did not show an increase expression. Therefore, perhaps BOR1 could be regulated under $\mathrm{B}$ toxicity, which was not observed in our experiments. However, the expression of the other genes (BOR2, BOR4 and BOR7;1) greatly increased after encapsulated B application. This was not directly related with $B$ concentration, as free $B$ also increased the concentration in leaves but did not produce an increase in BOR2, BOR4, and BOR7;1 expression. This was also observed with the NIPs (NIP1;2, NIP1;3; NIP4;1, NIP4;2, NIP5;1, NIP6;1, and NIP7;1), which only increased in expression with encapsulated B. Therefore, the results could be associated with the decreased mobility of free B into leaves, as compared with encapsulated $B$ [24]. Therefore, the encapsulated B applied to in vitro plants produced changes in the expression related to $B$, though only when $B$ was able to penetrate and be transported into the leaf tissues.

\section{Conclusions}

The results of this work support the high efficiency of encapsulated boron penetration and internal transport through leaf tissues as compared to free B application. This was associated by the increase in sugar metabolism (decrease in sucrose and increase in glucose 
and fructose), which could point to a connection with the primary metabolism for the subsequent changes provided by B transport into cells. The increase in NIP aquaporin expression involved in B transport and B transporters was associated with the B transport and mobilization within the cell, which should change according to a higher-than-optimal boron concentration, as the plants were not B-deficient. Thus, B storage could be the most plausible action that resulted in all the observed changes. In this sense, the fact that $B$ could interact with other micronutrient transporters, such as Fe and Mn, thus increasing their concentration in leaves, needs to be elucidated.

The result obtained with nanoencapsulation of B supports the investigation of nanotechniques that provide higher efficiency in foliar fertilization. The fact that nanovesicles provide a compatible and environmentally friendly system for ensuring precision fertilization opens a new area of investigation. Additionally, the fact that changes in metabolism and gene expression occurred with nanovesicles determines the need to further investigate the mechanism of action.

Supplementary Materials: The following are available online at https:/ /www.mdpi.com/article/ 10.3390/app12041788/s1, Table S1: Iddentification of important residues of I. batatas NIPs protein sequences derived from consensus sequences of I. triloba and I. nill. The specified residues presented are NPA motif, ar/R selectivity fliter and Froger's positions (FPs). Also included the residues of some sequences from $Z$. mays, $O$. sativa and $A$. thaliana previously described as capable of $B$ transport; Table S2: Gene names and its corresponding identificator from NCBI data base of I. triloba and I. nill NIP aquaporins isoforms.

Author Contributions: Conceptualization, M.C. and G.B. methodology, J.N.-E.; software, J.N.-E., G.B. and J.N.-E., formal analysis, G.B. and J.N.-E.; investigation, J.N.-E., P.G.-G., J.J.R., A.P.; resources, A.P., writing—original draft preparation, J.N.-E.; writing—review and editing, M.C.; supervision, M.C. and G.B.; project administration, M.C., funding acquisition, M.C. All authors have read and agreed to the published version of the manuscript.

Funding: This work was supported by the Spanish Ministry of Science and Innovation [RTC-20176544-2].

Acknowledgments: The authors thank Mario G. Fon for the correction of the editing language of the manuscript.

Conflicts of Interest: The authors declare that the research was conducted in the absence of any commercial or financial relationships that could be construed as a potential conflict of interest.

\section{References}

1. Nguyen, H.; Chen, C.-C.; Lin, K.-H.; Chao, P.-Y.; Lin, H.-H.; Huang, M.-Y. Bioactive Compounds, Antioxidants, and Health Benefits of Sweet Potato Leaves. Molecules 2021, 26, 1820. [CrossRef]

2. Bovell-Benjamin, A.C. Sweet Potato: A Review of its Past, Present, and Future Role in Human Nutrition. Adv. Food Nutr. Res. 2007, 52, 1-59.

3. Echer, F.R.; Dominato, J.C.; Creste, J.E.; Santos, D.H. Fertilization with boron and potassium on sweet potato nutrition and yield. Hortic. Bras. 2009, 27, 171-175. [CrossRef]

4. Smith, M.; Drew, R. Current Applications of Tissue Culture in Plant Propagation and Improvement. Funct. Plant Biol. 1990, 17, 267-289. [CrossRef]

5. Ruta, C.; Lambardi, M.; Ozudogru, E.A. Biobanking of vegetable genetic resources by in vitro conservation and cryopreservation. Biodivers. Conserv. 2020, 29, 3495-3532. [CrossRef]

6. Mycock, D.; Blakeway, F.; Watt, M.; Bornman, C. General applicability of in vitro storage technology to the conservation and maintenance of plant germplasm. S. Afr. J. Bot. 2004, 70, 31-36. [CrossRef]

7. Lambardi, M.; National, I.; Jain, S.M. Protocols for Micropropagation of Selected Economically-Important Horticultural Plants; Humana Press: Totowa, NJ, USA, 2013; Volume 994, ISBN 978-1-62703-073-1.

8. Kapilan, R.; Vaziri, M.; Zwiazek, J.J. Regulation of aquaporins in plants under stress. Biol. Res. 2018, 51, 4. [CrossRef]

9. Murata, K.; Mitsuoka, K.; Hirai, T.; Walz, T.; Agre, P.; Heymann, B.; Engel, A.K.; Fujiyoshi, Y. Structural determinants of water permeation through aquaporin-1. Nature 2000, 407, 599-605. [CrossRef]

10. Maurel, C.; Boursiac, Y.; Luu, D.T.; Santoni, V.; Shahzad, Z.; Verdoucq, L. Aquaporins in Plants. Physiol. Rev. 2015, 95, 1321-1358. [CrossRef] 
11. Sui, H.; Han, B.-G.; Lee, J.K.; Walian, P.J.; Jap, B.K. Structural basis of water-specific transport through the AQP1 water channel. Nature 2001, 414, 872-878. [CrossRef]

12. Froger, A.; Thomas, D.; Delamarche, C.; Tallur, B. Prediction of functional residues in water channels and related proteins. Protein Sci. 1998, 7, 1458-1468. [CrossRef]

13. Curticăpean, M.-C. Plant Aquaporins. Acta Biol. Marisiensis 2019, 2, 36-48. [CrossRef]

14. del Carmen Martinez-Ballesta, M.; Carvajal, M. New challenges in plant aquaporin biotechnology. Plant Sci. 2014, 217-218, 71-77. [CrossRef]

15. Lopez-Zaplana, A.; Nicolas-Espinosa, J.; Carvajal, M.; Bárzana, G. Genome-wide analysis of the aquaporin genes in melon (Cucumis melo L.). Sci. Rep. 2020, 10, 22240. [CrossRef]

16. Tanaka, M.; Fujiwara, T. Physiological roles and transport mechanisms of boron: Perspectives from plants. Pflügers Arch.-Eur. J. Physiol. 2008, 456, 671-677. [CrossRef]

17. Dannel, F.; Pfeffer, H.; Römheld, V. Characterization of root boron pools, boron uptake and boron translocation in sunflower using the stable isotopes $10 \mathrm{~B}$ and 11 B. Funct. Plant Biol. 2000, 27, 397-405. [CrossRef]

18. Poschenrieder, C.; Busoms, S.; Barceló, J. How Plants Handle Trivalent (+3) Elements. Int. J. Mol. Sci. 2019, 20, 3984. [CrossRef]

19. Kato, Y.; Miwa, K.; Takano, J.; Wada, M.; Fujiwara, T. Highly Boron Deficiency-Tolerant Plants Generated by Enhanced Expression of NIP5;1, a Boric Acid Channel. Plant Cell Physiol. 2009, 50, 58-66. [CrossRef]

20. Schnurbusch, T.; Hayes, J.; Hrmova, M.; Baumann, U.; Ramesh, S.; Tyerman, S.; Langridge, P.; Sutton, T. Boron Toxicity Tolerance in Barley through Reduced Expression of the Multifunctional Aquaporin HvNIP2;1. Plant Physiol. 2010, 153, 1706-1715. [CrossRef]

21. Hanaoka, H. Fujiwara Channel-mediated boron transport in rice. Plant Cell Physiol. 2007, 48, 844.

22. Du, W.; Pan, Z.-Y.; Hussain, S.B.; Han, Z.-X.; Peng, S.-A.; Liu, Y.-Z. Foliar Supplied Boron Can Be Transported to Roots as a Boron-Sucrose Complex via Phloem in Citrus Trees. Front. Plant Sci. 2020, 11, 250. [CrossRef]

23. Rios, J.J.; Yepes-Molina, L.; Martinez-Alonso, A.; Carvajal, M. Nanobiofertilization as a novel technology for highly efficient foliar application of Fe and B in almond trees. R. Soc. Open Sci. 2020, 7, 200905. [CrossRef]

24. Rios, J.J.; Lopez-Zaplana, A.; Bárzana, G.; Martinez-Alonso, A.; Carvajal, M. Foliar Application of Boron Nanoencapsulated in Almond Trees Allows B Movement Within Tree and Implements Water Uptake and Transport Involving Aquaporins. Front. Plant Sci. 2021, 12, 2373. [CrossRef]

25. Yepes-Molina, L.; Martínez-Ballesta, M.C.; Carvajal, M. Plant plasma membrane vesicles interaction with keratinocytes reveals their potential as carriers. J. Adv. Res. 2020, 23, 101-111. [CrossRef]

26. Rios, J.J.; Ibáñez, P.G.; Carvajal, M. The use of biovesicles to improve the efficiency of Zn foliar fertilization. Colloids Surf. B Biointerfaces 2019, 173, 899-905. [CrossRef]

27. Arnon, D.I. Copper enzymes in isolated chloroplasts. Polyphenoloxidase in Beta vulgaris. Plant Physiol. 1949, 24, 1-15. [CrossRef]

28. Choi, Y.H.; Kim, H.K.; Hazekamp, A.; Erkelens, C.; Lefeber, A.A.W.M.; Verpoorte, R. Metabolomic Differentiation of Cannabis sativa Cultivars Using 1H NMR Spectroscopy and Principal Component Analysis. J. Nat. Prod. 2004, 67, 953-957. [CrossRef]

29. Choi, Y.H.; Kim, H.K.; Linthorst, H.J.M.; Hollander, J.G.; Lefeber, A.W.M.; Erkelens, C.; Nuzillard, A.J.-M.; Verpoorte, R. NMR Metabolomics to Revisit the Tobacco Mosaic Virus Infection in Nicotiana tabacum Leaves. J. Nat. Prod. 2006, 69, 742-748. [CrossRef]

30. Wu, S.; Lau, K.H.; Cao, Q.; Hamilton, J.P.; Sun, H.; Zhou, C.; Eserman, L.; Gemenet, D.C.; Olukolu, B.A.; Wang, H.; et al. Genome sequences of two diploid wild relatives of cultivated sweetpotato reveal targets for genetic improvement. Nat. Commun. 2018, 9 , 4580. [CrossRef]

31. Isobe, S.; Shirasawa, K.; Hirakawa, H. Current status in whole genome sequencing and analysis of Ipomoea spp. Plant Cell Rep. 2019, 38, 1365-1371. [CrossRef]

32. GuoLiang, L.; Guochun, X.; Zhaomiao, L.; Huawei, L.; Zhonghua, L.; Yongqing, X.; Hong, Z.; Rongchang, J.; Wenbin, L.; Yongxiang, Q.; et al. Selection of suitable reference genes for RT-qPCR normalisation in sweet potato (Ipomoea batatas L.) under different stresses. J. Hortic. Sci. Biotechnol. 2020, 96, 209-219. [CrossRef]

33. Ramakers, C.; Ruijter, J.M.; Deprez, R.H.L.; Moorman, A.F.M. Assumption-free analysis of quantitative real-time polymerase chain reaction (PCR) data. Neurosci. Lett. 2003, 339, 62-66. [CrossRef]

34. Vandesompele, J.; De Preter, K.; Pattyn, F.; Poppe, B.; Van Roy, N.; De Paepe, A.; Speleman, F. Accurate normalization of real-time quantitative RT-PCR data by geometric averaging of multiple internal control genes. Genome Biol. 2002, 3, research0034. [CrossRef] [PubMed]

35. Schmittgen, T.D.; Livak, K.J. Analyzing real-time PCR data by the comparative $C_{\mathrm{T}}$ method. Nat. Protoc. 2008, 3, 1101-1108. [CrossRef] [PubMed]

36. Brown, P.H.; Bellaloui, N.; Wimmer, M.A.; Bassil, E.S.; Ruiz, J.; Hu, H.; Pfeffer, H.; Dannel, F.; Römheld, V. Boron in Plant Biology. Plant Biol. 2002, 4, 205-223. [CrossRef]

37. Zahedi, S.M.; Karimi, M.; Da Silva, J.A.T. The use of nanotechnology to increase quality and yield of fruit crops. J. Sci. Food Agric. 2020, 100, 25-31. [CrossRef]

38. Barrios, A.C.; Rico, C.; Trujillo-Reyes, J.; Medina-Velo, I.A.; Peralta-Videa, J.R.; Gardea-Torresdey, J.L. Effects of uncoated and citric acid coated cerium oxide nanoparticles, bulk cerium oxide, cerium acetate, and citric acid on tomato plants. Sci. Total Environ. 2016, 563-564, 956-964. [CrossRef]

39. Cakmak, I.; Kutman, U.B. Agronomic biofortification of cereals with zinc: A review. Eur. J. Soil Sci. 2018, 69, 172-180. [CrossRef] 
40. Hao, B.; Ma, J.; Jiang, L.; Wang, X.; Bai, Y.; Zhou, C.; Ren, S.; Li, C.; Wang, Z. Effects of foliar application of micronutrients on concentration and bioavailability of zinc and iron in wheat landraces and cultivars. Sci. Rep. 2021, 11, 22782. [CrossRef]

41. Connolly, E.L.; Fett, J.; Guerinot, M.L. Expression of the IRT1 Metal Transporter Is Controlled by Metals at the Levels of Transcript and Protein Accumulation. Plant Cell 2002, 14, 1347-1357. [CrossRef]

42. Barberon, M.; Zelazny, E.; Robert, S.; Conéjéro, G.; Curie, C.; Friml, J.; Vert, G. Monoubiquitin-dependent endocytosis of the IRON-REGULATED TRANSPORTER 1 (IRT1) transporter controls iron uptake in plants. Proc. Natl. Acad. Sci. USA 2011, 108, E450-E458. [CrossRef] [PubMed]

43. Takano, J.; Wada, M.; Ludewig, U.; Schaaf, G.; von Wirén, N.; Fujiwara, T. The Arabidopsis Major Intrinsic Protein NIP5;1 Is Essential for Efficient Boron Uptake and Plant Development under Boron Limitation. Plant Cell 2006, 18, 1498-1509. [CrossRef] [PubMed]

44. Tanaka, M.; Takano, J.; Chiba, Y.; Lombardo, F.; Ogasawara, Y.; Onouchi, H.; Naito, S.; Fujiwara, T. Boron-Dependent Degradation ofNIP5;1mRNA for Acclimation to Excess Boron Conditions inArabidopsis. Plant Cell 2011, 23, 3547-3559. [CrossRef] [PubMed]

45. Sasaki, A.; Yamaji, N.; Yokosho, K.; Ma, J.F. Nramp5 Is a Major Transporter Responsible for Manganese and Cadmium Uptake in Rice. Plant Cell 2012, 24, 2155-2167. [CrossRef] [PubMed]

46. Yamaji, N.; Sasaki, A.; Xia, J.X.; Yokosho, K.; Ma, J.F. A node-based switch for preferential distribution of manganese in rice. Nat. Commun. 2013, 4, 2442. [CrossRef] [PubMed]

47. Bariya, H.; Bagtharia, S.; Patel, A. Boron: A Promising Nutrient for Increasing Growth and Yield of Plants. Plant Ecophysiol. 2014, 10, 153-170. [CrossRef]

48. Nasef, M.A.; Badran, N.M.; Abd El-Hamide, A.F. Response of peanut to foliar spray with boron and/or rhizobium inoculation. J. Appl. Sci. Res. 2006, 2, 1330-1337.

49. Lanquar, V.; Ramos, M.S.; Lelièvre, F.; Barbier-Brygoo, H.; Krieger-Liszkay, A.; Kraemer, U.; Thomine, S. Export of Vacuolar Manganese by AtNRAMP3 and AtNRAMP4 Is Required for Optimal Photosynthesis and Growth under Manganese Deficiency. Plant Physiol. 2010, 152, 1986-1999. [CrossRef]

50. Che, J.; Yamaji, N.; Ma, J.F. Efficient and flexible uptake system for mineral elements in plants. New Phytol. 2018, 219, 513-517. [CrossRef]

51. Igamberdiev, A.; Rodionova, M. Effect of glycolate pathway intermediates on succinate metabolization in maize and wheat leaves incubated in the dark. Fiziol. Rastenij 1992, 39, 126-134.

52. Zhang, Z.; Huang, J.; Gao, Y.; Liu, Y.; Li, J.; Zhou, X.; Yao, C.; Wang, Z.; Sun, Z.; Zhang, Y. Suppressed ABA signal transduction in the spike promotes sucrose use in the stem and reduces grain number in wheat under water stress. J. Exp. Bot. 2020, 71, 7241-7256. [CrossRef] [PubMed]

53. Du, X.; Zhang, X.; Xi, M.; Kong, L. Split application enhances sweetpotato starch production by regulating the conversion of sucrose to starch under reduced nitrogen supply. Plant Physiol. Biochem. 2020, 151, 743-750. [CrossRef] [PubMed]

54. Pommerrenig, B.; Diehn, T.A.; Bienert, G.P. Metalloido-porins: Essentiality of Nodulin 26-like intrinsic proteins in metalloid transport. Plant Sci. 2015, 238, 212-227. [CrossRef] [PubMed]

55. Porquet, A.; Filella, M. Structural Evidence of the Similarity of $\mathrm{Sb}(\mathrm{OH})_{3}$ and $\mathrm{As}(\mathrm{OH})_{3}$ with Glycerol: Implications for Their Uptake. Chem. Res. Toxicol. 2007, 20, 1269-1276. [CrossRef]

56. Tanaka, M.; Wallace, I.S.; Takano, J.; Roberts, D.M.; Fujiwara, T. NIP6;1 Is a Boric Acid Channel for Preferential Transport of Boron to Growing Shoot Tissues inArabidopsis. Plant Cell 2008, 20, 2860-2875. [CrossRef] 